\title{
Endosymbiont Bacteria Holospora undulata Confers Oxidative Tolerance in Host Paramecium caudatum
}

\author{
Rainey A. Stewart, Department of Biology, Indiana University - Bloomington \\ Faculty Mentor: Dr. Thomas Doak
}

\begin{abstract}
Daramecium is a genus of ciliated protozoan that, while unicellular, has a complex intracellular structure, comparable 1 to Metazoan cells, which has made them excellent models for the study of genetics and cellular functions. Holospora undulata is a bacterial endosymbiont specific to the species Paramecium caudatum; they are unable to grow outside of $P$. caudatum. The presence of this endosymbiont has proven to have an effect on the subsequent gene expression and cellular maintenance of its host cells. Recent studies have demonstrated that infection by H. obtusa increases the expression of host heat-shock genes and leads to both resistance at normally-lethal high temperatures and heat resistance in ciliary movement (Fujishima, Kawai, \& Yamamoto, 2005; Hori \& Fujishima, 2003). Heat-shock resistance occurs because bacterial DNA triggers the upregulation of its $P$. caudatum host's heat-shock genes (i.e., hsp60 and hsp70), although the mechanisms are not known (Hori \& Fujishima, 2003). These studies demonstrate that infection of P. caudatum by H. undulata (a closelyrelated species to $H$. obtusa) induces heat-shock resistance, but fail to address whether $H$. undulata protects against other common environmental stressors such as oxidative damage. To determine if infection by $H$. undulata has the ability to induce additional tolerances, we examined differences in oxidative tolerance, based on percent survival, between P. caudatum with and without $H$. undulata infection. Samples of both lines were treated with increasing concentrations of hydrogen peroxide, the number of surviving cells were counted, and the percent survivability of each sample was calculated. There was an approximate $20 \%$ increase in survival when $P$. caudautum was infected with $H$. undulata-thus $H$. undulata infections confer oxidative tolerance. Further studies will be conducted to determine if an increase in survivability occurs in response to other damaging mechanisms. Future work will also investigate if the genes responsible for oxidative damage repair are upregulated, in addition to the already characterized heat-shock genes.
\end{abstract}

KEYWORDS: Paramecium caudatum, oxidative tolerance, endosymbiont, host, heat-shock

\section{AUTHOR INFORMATION}

All correspondence should be sent to rainstew@indiana.edu.

\section{ACKNOWLEDGMENTS}

Special thanks to Michael Lynch for use of the Lynch Lab and Thomas Doak for his mentorship and support with the research. Additional thanks to Lydia Bright for her expertise. This research was funded by NSF MCB-1518060: Evolution of the Transcriptional Vocabulary: The Paramecium aurelia Complex.

\section{REFERENCES}

Hori, M., \& Fujishima, M. (2003). The endosymbiotic bacterium Holospora obtusa enhances heat-shock gene expression of the host Paramecium caudatum. The Journal of Eukaryotic Microbiology, 50(4), 293-298.

Fujishima, M., Kawai, M., \& Yamamoto, R. (2005). Paramecium caudatum acquires heat-shock resistance in ciliary movement by infection with the endonuclear symbiotic bacterium Holospora obtusa. FEMS Microbiology Letters, 243(1), 101-105. 